\title{
Environmental risk management. Forecasting and modeling of emergency risk management situations
}

\author{
Vitaly Kas'yanov ${ }^{1}$, Vadim Danilchenko ${ }^{1, *}$, Vasily Amelin ${ }^{2}$ and Valeria Tolmacheva ${ }^{2}$ \\ ${ }^{1}$ Moscow State University of Civil Engineering, Yaroslavskoe shosse, 26, Moscow, 129337, Russia \\ ${ }^{2}$ South-West State University, 305040, $50^{\text {th }}$ year of October street, 94, Kursk, Russia
}

\begin{abstract}
For processes of environmental risk management, the results of a study of its perception are important. The identified priorities in the public concern about the state of the environment should be taken into account when preparing the necessary environmental measures. The prevention of risk or its reduction should take into account not only quantitative, but also qualitative characteristics of risk, which are caused by various factors and mechanisms of risk perception. Risk perception research data is essential for adequate risk communication, so managers involved in risk management should be interested in increasing the use of such data.
\end{abstract}

\section{Introduction}

People manage risk for nearly four thousand years. It is known that approximately 3,900 years ago property insurance was already carried out in ancient Mesopotamia. The law of the king Hamurappi, dating back to $1950 \mathrm{BC}$, contained rules for granting loans on the security of a ship, which provided for insurance risk and payment of the appropriate amount in the event of the vessel's loss and loss of cargo. This type of insurance was developed later in ancient Greece. The first insurance policy, insuring human life, appeared much later - in 1583 in England. The first legislative act aimed at reducing environmental risk can be considered the edict of the English King Edward I, signed by him more than seven hundred years ago, in 1285. This decree forbade burning in so-called "soft" coal, in which a lot of air polluting impurities.

In order to prevent or reduce the risk of numerous and diverse documents, the scope of which may be limited to any one enterprise, and may apply to the whole country. Such documents include legislation and regulations aimed at protecting health, improving working conditions, reducing pollution of the environment, ensuring safety on the roads, standardizing the quality of goods sold, etc. [1]

\footnotetext{
* Corresponding author: vadim.dkr@mail.ru
} 


\section{Methodology}

The use of risk parameters in the legislation requires an accurate quantitative determination of the two most important concepts - the maximum allowable risk and negligible (absolutely acceptable) risk. A risk is deemed to be negligible if its level due to its smallness cannot be reliably detected against the background of already existing risks. In most countries of Western Europe, the individual risk to which the population is exposed (and not to the production personnel) is considered negligible if its level does not exceed 10-6 per year. The exception is the Netherlands, where a value of 10-6 per year is considered the maximum permissible risk, and a negligible risk is fixed at the level of 10-8 year. [2] In the United States, an individual risk tolerance of 10-6 is set not for one year, but for the entire life of a person, the average duration of which is assumed to be 70 years. Consequently, the annual individual risk tolerance in the United States amounts to 10-6 / 70 $=1.43 \cdot 10^{\wedge} 8$ year 1 .

It should be noted that the values of individual risk given are theoretical. Practical values of permissible individual risks can be much higher. For example, the US Supreme Court set the lower limit for significant individual risk due to the presence of carcinogens in the environment, equal to $1 \cdot 10-3$. Therefore, in this case, any individual risk less than 1 . 10-3 should be considered insignificant. According to the regulations of the US Environmental Protection Agency, the acceptable (acceptable) risk from substances with carcinogenic properties lies in the range from 10-4 to 10-6.

The upper limit of permissible risk (the maximum permissible risk) is different for the population and personnel working in hazardous conditions. In Russia, the maximum permissible individual risk for technogenic exposure of persons from staff is assumed to be $1.0 \cdot 10^{\wedge} 3$ per year, and for the population $-5.0 \cdot 10^{\wedge} 5$ per year (the latter value is 50 times higher Russian Federation is assumed to be 106 per year).

Values of acceptable risk are used as criteria in the process of environmental risk management. The purpose of this process is to reduce the level of risk to an acceptable one. The risk management process is based on the results of quantitative risk assessment, which allows[3-5]

- compare alternative projects of potentially dangerous objects and technologies

- identify the most dangerous risk factors acting on the site

- create databases and knowledge bases for expert systems to support technical decisions and the development of regulatory documents

- identify priority areas for investments aimed at reducing risk and reducing risk.

First, a comparison of the risk assessment results for the situation under consideration and the corresponding criteria is carried out. After this comparison, risk reduction options are found, each of which is evaluated taking into account the costs of its implementation. Evaluation of options is an iterative operation, it is repeated until an optimal solution is chosen. [4]

An essential step in the search for risk reduction options (see Figure 7.3) is the prediction of changes in the parameters of the current situation and modeling the behavior of the object in question. A scientific forecast is a statement in the form of a probabilistic statement about the behavior of a certain system in the future, based on studying and summarizing past experience using intuitive ideas about the development of this system in the future. Scientific forecasts are made by experts - experts in the field in question. [6] The basis of the forecasted expertise is a special scientific discipline - prognostics. Often, instead of the term "scientific forecast", the term "expert assessments" is used. [7]

The essence of the method of expert assessments lies in the fact that specialists are offered to answer questions about the future behavior of objects or systems characterized by uncertain parameters or unexplored properties. Expert estimates are made in the form of 
qualitative characteristics or quantitative values of the probabilities of the considered events or processes related to a certain period of time. At the same time, importance is attached to the formation of the rating scale used by experts. It was established that the optimal rating scale should have a relatively small number of gradations (from 3 to 8 ), each gradation is assigned a certain probability interval or a certain probability value. In addition, each graduation should be accompanied by a brief qualitative characteristic (verbal or linguistic explanation)[8].

Methods of expert estimates using probabilities are part of a probabilistic analysis of the safety of technological objects with unpredictable behavior due to unknown values of the factors determining this behavior. Probabilistic safety analysis can cover dozens and hundreds of different scenarios (for example, using the tree method), but it can also be limited to considering single events or processes. [4,9]

Currently, there are several dozen expert assessment methods, the most famous of which is the collective discussion and coordination using the Delphi method. We can say that the creators of the method of expert assessments were the Delphic oracles, that is, the priests of the Temple of Apollo at the foot of Parnassus Mountain in Greece. Their prediction of a particular event in ancient Greece was communicated to the people only after all the members of the council of sages familiarized themselves with all the circumstances of the case and discussed them from all sides $[4,9,10,11]$.

The adoption of expert decisions on the Delphi method is carried out in the following order:

1. Formation of a group of experts - major specialists in the area in which the problem is located.

2. Initial filling in by the experts of the prepared questionnaires, accompanied by providing them with all the available information on the problem (first round);

3. Processing of questionnaires and a written statement of its main results.

4. Acquaintance of experts with the results of processing the questionnaires and the refilling of similar sheets (second round) with the indication that the same questions should be given new answers taking into account the results of the first round. There may be two or more such tours, depending on the degree of consistency of the responses.

The Delphi method was used, in particular, when analyzing possible violations of the integrity of tanks in a radioactive waste storage facility at the Hanford Nuclear Center. Each of the numerous scenarios for the occurrence of an emergency during a given time interval was characterized by experts in one of three grades of the rating scale with the corresponding interval values of the probability of the situation:

1. "It seems possible, can be considered as foreseeable within reasonable limits" (reasonably foreseeable): probability $\mathrm{P}>10-2$.

2. "Very unlikely": $10-4<\mathrm{P}<10-2$.

3. "Extremely improbable" (extremely unlikely): $\mathrm{P}<10-4$.

Thus, the method of expert assessments is used to solve problems associated with risk management (for example, planning systems to ensure the technological, environmental and social safety of a certain object) in cases where a rigorous calculation is impossible due to the presence of fundamental uncertainties. Below are examples of its specific use in combination with another method called the tree method (see Chap. 2). This method is widely used in making risk-related decisions. Among its advantages are the convenience and clarity of graphical representation, as well as a significant ease of calculations on computers. The tree method is especially effective in cases where a complex problem can be divided into one or another number of relatively simple tasks, each of which is solved separately, after which a unique synthesis of a complex solution is performed. In the process of forecasting emergencies and their modeling, using the tree method allows one to calculate the probability of the implementation of a specific scenario involving several 
events. The tree structure is based on the basic theorems of probability theory the addition theorem and the multiplication theorem $[12,13]$.

\section{Results and Discussion}

In the process of quantitative risk assessment and management, significant difficulties are caused by the presence of uncertainties in the reliability characteristics of personnel employed at potentially hazardous facilities. Man-made disasters such as the explosion of a nuclear reactor at the Chernobyl nuclear power plant or the leakage of toxic gases at a pesticide plant in Bhopal (India) have shown that it is not possible to solve the risk reduction problem using purely engineering, technological or organizational methods. This is largely due to the fact that in such emergency situations there are not foreseen scenarios for the development of events in which the response of the staff is inadequate, as a result of which erroneous actions are performed. An analysis of about 30 thousand incidents at nuclear power facilities in the United States showed that in about half of them a unique combination of technological failures and human error was formed. Expansion of the scope of application of automated tools leads to new problems, as new types of failures and errors appear. Computerization leads to dangerous software-related errors. In addition, under these conditions, the whole complex of relations between a person, on the one hand, and a machine or computer, on the other, changes in an unpredictable way. Studies performed in economically developed countries show the need for a comprehensive study of the role of the human factor in risk-related technologies and at potentially dangerous objects.

Over the past two decades, methods for quantitative assessment of human reliability have changed significantly; now they differ sharply from the approaches traditionally used in calculating equipment reliability indicators. To study the human factor, special technical tools are created simulating human-machine interaction complexes, simulation installations and research simulators. They are used for a comprehensive study of personnel actions, analysis of the behavior of operators, and identification of major errors. One of the directions of studying the role of the human factor is to identify the causes of erroneous actions of people serving complex technological installations. To determine the characteristics of errors that are different in nature, psychologists develop their classification. One of these classifications was proposed in 1990 by Reason in his book Human Errors [14].

The above classification is used in the simulation of human interaction with the machine. All dangerous actions that can cause a man-made emergency or catastrophe can be divided into unintentional and intentional. The first of them, in turn, are divided into errors and omissions, and the second - on errors and violations. The failures are caused by lack of attention (for example, the order of performing two consecutive operations is confused), while the reasons for omissions are memory deficiencies (for example, the operator has forgotten about one link in the chain of necessary operations). The reasons for missteps can be incorrect execution of existing rules (for example, incorrect fulfillment of the rule necessary in a given situation, or an action according to such a rule that is generally not applicable in the prevailing situation) or insufficient knowledge of actions in both normal and emergency situations. Violations are conscious actions leading to deviations from the normal functioning of the object.

Human factor modelling has become an integral part of probabilistic safety analysis (PSA) of potentially hazardous objects. This part of the PSA is the most complex, it allows to take into account only relatively simple personnel errors. A serious problem is the recording of personnel actions under stressful conditions of an accident with an inevitable shortage of time. Complex errors, the number of which can be quite large, are very difficult 
to model, and multiple errors (such as those made at the Chernobyl nuclear power plant) are practically impossible to analyse at all [15].

Despite the creation of modern models that allow, within certain limits, to describe the interaction of the operator with the machine, the problems caused by the role of the human factor are still far from solution. The urgency of these problems has led to the emergence of a new branch of knowledge - safety culture. The term "safety culture" was introduced in 1986 by experts from the International Advisory Group on Nuclear Safety (ISOL) of the International Atomic Energy Agency (IAEA) in the final document on the causes and consequences of the Chernobyl accident. In a subsequent document of the IAEA IAEA "Basic Principles for the Safety of Nuclear Power Plants", published in 1990, the safety culture was described as a "fundamental management principle". According to the definition adopted by the IAEA, safety culture is a set of characteristics and characteristics of organizations and individuals, which establishes that the safety problems of a nuclear facility as having the highest priority is given attention, determined by their importance. Subsequently, the definition of a safety culture was extended to any potentially hazardous objects and high-risk technology. Thus, by definition of Merritt-Helmreich (1996), a safety culture is more than just a group of individuals who follow a set of rules for the safe conduct of work; This is a group of people who, in their behavior, are guided by the general confidence in the importance of ensuring security and understand the need for each team member to be willing to support the norms of collective security and help other team members to strive for this common goal.

Environmental risk management is carried out through the development and application of legal acts that establish environmental legal responsibility. In Russia (more precisely, in the former USSR) the concept of environmental legal liability was first formulated in the RSFSR Law "On Enterprises and Business", which provided for compensation for damage from pollution and the irrational use of the natural environment [4]. Then this provision was developed in a special Law of the RSFSR "On the Protection of the Environment", where, in particular, three types of damage were established that are subject to compensation:

-damage caused to the environment by a source of increased danger;

-damage caused to the health of citizens by adverse effects on the environment;

-damage to property of citizens[16].

The Law of the Russian Federation "On the Industrial Safety of Hazardous Production Facilities", adopted in 1997, stipulates that an enterprise that is a source of increased danger is obliged to provide measures to protect the population and the environment from dangerous influences. This law also introduces a procedure for licensing hazardous production facilities and considers the possibility of revocation or suspension of a license in the event of non-compliance with industrial safety requirements or non-compliance with accepted standards. In addition, for the first time in this law, Russia introduced compulsory environmental insurance, which is a liability insurance for causing harm (for example, accidental pollution of the environment) when operating a hazardous production facility. The minimum amount of insurance liability of enterprises is determined depending on the hazard level of production. The law establishes that for the most hazardous production facilities the amount of the insurance amount cannot be less than 70,000 minimum wages (MW) established by the legislation of the Russian Federation on the day of the conclusion of the insurance contract. Environmental insurance should be considered an important part of the environmental risk management mechanism. [17]

According to modern requirements, the developed programs to reduce environmental risks should provide for carefully calibrated estimates of the necessary costs. At the same time it is necessary to determine the priority directions of spending funds Criteria for choosing priorities may vary. Thus, the US Budget Act for 1996 allocated the Department 
of Energy \$ 6.5 billion in expenditures to improve the environment, and the lion's share of this amount - 5.1 billion - was earmarked for measures to reduce environmental risk. In justifying its financial needs, the Energy Department presented qualitative criteria for assessing environmental risks, distributing them to high, medium and low.

Nowadays, the view that quantitative criteria for identifying priorities should be used is becoming increasingly common. The latter means that risk management is carried out according to a scheme that takes into account the categories of its two components - the probability of a dangerous event $\mathrm{P}$ and its consequences $\mathrm{Q}$. For this, a certain number of categories of probability and consequences are considered, and each category is assigned a certain rating [18].

First, the probability and consequences of this hazardous event are divided into five categories, each of which is characterized by the following qualitative characteristics: minimum, low, medium, high and maximum. Then these categories are assigned ratings from 1 to 5 . The risk values $\mathrm{R}$ as a product of PQ are also divided conditionally into five categories, for example, as follows:

maximum risk $\mathrm{R}=\mathrm{PQ}>20$,

high risk $15<\mathrm{R}<20$,

average risk $10<\mathrm{R}<15$,

low risk $5<\mathrm{R}<10$,

minimum risk $\mathrm{R}<5$.

In such a view, maximum and high risks are usually considered unacceptable, medium and low risks $\mathrm{R}$ are limited to acceptable, and minimal risk is considered to be absolutely acceptable. The value of the considered scheme is that, depending on the magnitude of the risks, they can be prioritized, that is, arranged in order. This is necessary to prioritize environmental protection measures and appropriate allocation of funds for their implementation (investments). [4, 9, 17]

The considered principle is applied, in particular, by the US Department of Defense to prioritize environmental projects and optimize environmental expenditures. As an example, one can cite the methodology used at bases and in units of the United States Air Force [18]. This methodology uses a factor table for quantitative assessments of environmental risks, which considers five categories of event probability and four categories of its consequences.

It is seen that the rows of the table characterize the categories of severity of the consequences of adverse events, and its columns attribute quantitative estimates (quantify) the categories of probability (frequency) of such events. Regulatory documents of the USAF contain explanations of both types of these categories, which are as follows.

Catastrophic are consequences that are characterized by a complete disruption of the facility's operation, a complete failure of its systems, material losses amounting to more than $\$ 1$ million, the presence of deaths or serious injuries among personnel, or irreversible damage to the environment, accompanied by a violation of environmental legislation. Critical are the consequences, characterized by a significant violation of the functions of the object, failure of the main nodes of its systems, material losses amounting to more than 200 thousand but less than $\$ 1$ million, the appearance of permanent disability, serious injuries or occupational diseases in at least three people from the staff or damage to the habitat caused by reversible damage that caused a violation of environmental legislation. The insignificant (marginal) effects include those characterized by an insignificant disruption of the object's operation, minor damage to its systems, material losses amounting to more than 10 thousand but less than 200 thousand dollars, the appearance of a minor injury or occupational disease resulting in the loss of one working day. habitat recoverable damage, 
not accompanied by violation of environmental legislation. The consequences, characterized by very insignificant violation of the object's functions, insignificant damage to its systems, material losses amounting to more than 2 thousand but less than 10 thousand dollars, the occurrence of such minor injuries or occupational diseases that did not lead to the loss of even one working day, are considered to be negligible. or inflicted habitat with minimal reparable damage that is not accompanied by violation of environmental legislation $[19,20]$.

\section{Conclusions}

To characterize the categories of probability (frequency) of environmentally adverse events, it is considered expedient to use not only quantitative, but also qualitative signs.

For example, an exceptionally high level of risk can be identified for the catastrophic consequences of a probable event (a factor is 2) or for the critical consequences of a frequent event (a factor is 3 ).

The methodology for prioritizing environmental projects, adopted by the US Air Force, has only recently begun to be put into practice, but it has already managed to establish itself on the positive side. In the United States, it is set as an example to other departments, which are faced with the task of developing legal documents designed to regulate environmental activities.

\section{References}

1. Bartell S.M. Ecological/Environmental Risk Assessment // Risk Assessment and Management Handbook. New York, 1996. P. 10.3-10.59.

2. Chicken J.C. Risk Handbook. London, 1996. 310 p.

3. Chicken J.C., Harbison S.A. Differences Between Industries in the Definition of Acceptable Risk // New Risks. New York, 1990. P. 123-128.

4. Borkovskaya V.G. Complex models of active control systems at the modern developing enterprises // Advanced Materials Research. 2014. T. 945-949. C. 30123015.

5. Borkovskaya V.G. Environmental and economic model life cycle of buildings based on the concept of "green building" // Applied Mechanics and Materials. 2014. T. 467. C. 287-290.

6. Cohen B.L. Catalog of Risks Extended and Updated // Health Physics. 1991. Vol. 61. P. 89-96.

7. Borkovskaya V.G. Post bifurcations of the concept of the sustainable development in construction business and education // Advanced Materials Research. 2014. T. 860863. C. 3009-3012.

8. Cohen B.L. The Nuclear Energy Option. An Alternative for the 90s. New York, 1990. $320 \mathrm{p}$.

9. Borkovskaya V.G. Project risks. Scientific Review number 23, 2015. Moscow. 212214pp.

10. Covello V.T. Communications Risk in Crisis and Noncrisis Situations // Risk Assessment and Management Handbook. For Environmental, Health, and Safety Professionals. New York, 1996. P. 45-65.

11. Borkovskaya V, Passmore D. Application of Failure Mode and Effects Analysis in Ecology in Russia. MATEC Web of Conf., 193 (2018) 05027. DOI: https://doi.org/10.1051/matecconf/201819305026 
12. Covello V.T., Sandman P.T., Slovic P. Guidelines for Communicating Information about Chemical Risks Effectively and Responsibly // Acceptable Evidence. Science and Values in Risk Management. New York, 1991. P. 66-90.

13. Borkovskaya V.G., Passmore D. Behavioral engineering model to identify risks of losses in the construction industry. Advances in Economics, Business and Management Research (France-Netherlands). Atlantis Press. In press.

14. Dzuray E.J, Maranto A.R. Assessing the Status of Risk-Based Approaches for the Prioritization of Federal Environmental Spending // Federal Facilities Environmental J. 1999. N 5. <http://www.research.umbc.edu/ maranto/fedfac.htm>.

15. Environmental Encyclopedia. Detroit, 1994. 110 p.

16. Goedkoop M. The Eco-indicator 95. Final Report. Utrecht, The Netherlands. 1995. http://www.pre.nl/eco-indicator95/ei-95-reports.htm

17. Borkovskaya V.G, Bardenwerper W, Roe R. Sustainability Risk Management: The Case for Using Interactive Methodologies for Teaching, Training and Practice in Environmental Engineering and Other Fields. Advances in Economics, Business and Management Research (France-Netherlands). Atlantis Press. In press.

18. Goedkoop M., Spriensma R. The Eco-indicator 99. A damage oriented method for Life Cycle Impact Assessment. Methodology Report. Amersfoort, The Netherlands, 2000. <http://www.pre.nl/download/EI99_methodology_v2.pdf>

19. Hallenbeck W.H. Quantitative Risk Assessment for Environmental and Occupa-tional Health. Boca-Raton, 1993. 212 p.

20. Borkovskaya V.G., Belikova E.S. Risks and the actual state of the quality management system at enterprises of the construction industry. International scientific and technical journal "Real Estate: Economics, Management". Economics and management of real estate. Moscow. 4-2017. P39-P43 